\title{
Design of layered transformation-optics devices of arbitrary shape
}

\author{
Bogdan-Ioan Popa* and Steven A. Cummer ${ }^{\dagger}$ \\ Department of Electrical and Computer Engineering, Duke University, Durham, North Carolina 27708, USA
}

(Received 15 July 2010; published 30 September 2010)

\begin{abstract}
Transformation-optics devices of arbitrary shapes usually result in material parameters inside the device that feature level sets of different shapes. Consequently, these devices cannot easily be implemented using a layered architecture and thus are, generally, more difficult to realize in practice. We present a method of designing two-dimensional transformation-optics devices of arbitrary shapes characterized by material parameters of same-level sets, thus suitable to be implemented through concentric layers, each layer being made of a single type of material or metamaterial. Remarkably, we show that transformations leading to such designs are obtained from solutions to the well-known eikonal equation. This approach allows fabrication techniques developed for cylindrical designs of circular cross section to be directly applied to devices of other shapes.
\end{abstract}

DOI: 10.1103/PhysRevA.82.033837

PACS number(s): 42.70.-a, 42.25.Fx, 41.20.Jb

\section{INTRODUCTION}

Transformation optics is a design technique that gives the freedom to manipulate electromagnetic waves in ways thought until recently impossible [1,2]. One challenge in transformation optics is that it specifies materials that are anisotropic and inhomogeneous and consequently difficult to realize. For the simple cylindrical and spherical device geometries extensively analyzed in the past (see [1-9] for a short list), the inhomogeneity is such that all material parameters have only radial variation, which makes these devices considerably easier to implement in layers. Schurig et al. [3] showed how this can be conceptually done for the scattering-reducing coating (also called "invisibility cloak" or, simply, "cloak"). The layered architecture also allows advanced design techniques, such as optimization methods $[10,11]$, that can significantly simplify the design in order to make it easier to realize.

Unlike the special geometries mentioned earlier, for arbitrary transformation-optics devices considered in the literature (see for example [12-15]), the components of the material parameter tensors are constant on different contours and therefore are much harder to implement when anisotropy is also required (the vast majority of cases). As an example of such a device that cannot be realized through layers, we consider the invisibility cloak analyzed by Rahm et al. [12]. The device consists of a cylindrical shell of square cross section surrounding a perfectly conducting square object. Central to the transformation-optics method is a set of functions called transformation (or mapping) functions that determine the geometry and composition of the final device. The natural choice of transformation functions used in [12] produces a device whose material parameters in the principal coordinate systems (i.e., the systems in which the permittivity and permeability tensors are diagonal) are shown in Fig. 1. We notice that $\epsilon_{11}$ and $\epsilon_{22}$ have parabolic contours of constant values (also called level sets), while $\epsilon_{33}$ has linear level sets. This feature makes the design unpractical to implement using an easier-to-realize layered architecture.

\footnotetext{
*bap7@ee.duke.edu

†cummer@ee.duke.edu
}

Fortunately, transformation optics is a general technique that allows us to choose the transformation from a relatively large pool of transformation functions. In this article we show how to determine the proper transformations that provide devices for which all the material parameters have level sets of identical shape and therefore can be realized in a layered architecture. We call these devices "layered devices."

We note that, although each level set is characterized by constant material parameters, the orientation of the principal system can vary within each level set, as illustrated by the realization of the cylindrical cloak [3]. More specifically, in this cylindrical cloak case the orientation of the principal system was such that one of its axes was always tangent to the corresponding circular level set.

Remarkably, the transformation functions obtained through our method are derived from solutions to the wellknown eikonal equation extensively used in optics, which allows an interesting parallelism between propagation of optical wave fronts and how the transformation functions are generated. Together with optimization techniques discussed in [10], our approach provides a practical way to design realizable arbitrary-shaped transformation-optics devices.

We focus on two-dimensional (2D) devices of arbitrary shape. We start by analyzing a general set of arbitrary transformations and the constraints it has to satisfy in order to obtain layered devices. Based on these constraints, we choose a family of transformation functions that give material parameters of same-level sets. In the end we illustrate our approach in an example, by designing a layered invisibility cloak of same geometry as that considered in [12] and presented in Fig. 1.

\section{METHOD TO OBTAIN LAYERED TRANSFORMATION-OPTICS DEVICES}

We consider the transformation framework presented in Fig. 2. We wish to manipulate the electromagnetic fields inside the $\Gamma_{b}^{r}$ curve in the real space represented by the $(x, y, z)$ coordinate system in order to obtain fields outside $\Gamma_{b}^{r}$ that have the desired behavior specified in the $(u, v, w)$ virtual space. For this purpose we need to find a set of smooth mapping (or transformation) functions $u(x, y, z), v(x, y, z)$, and $w(x, y, z)$ 


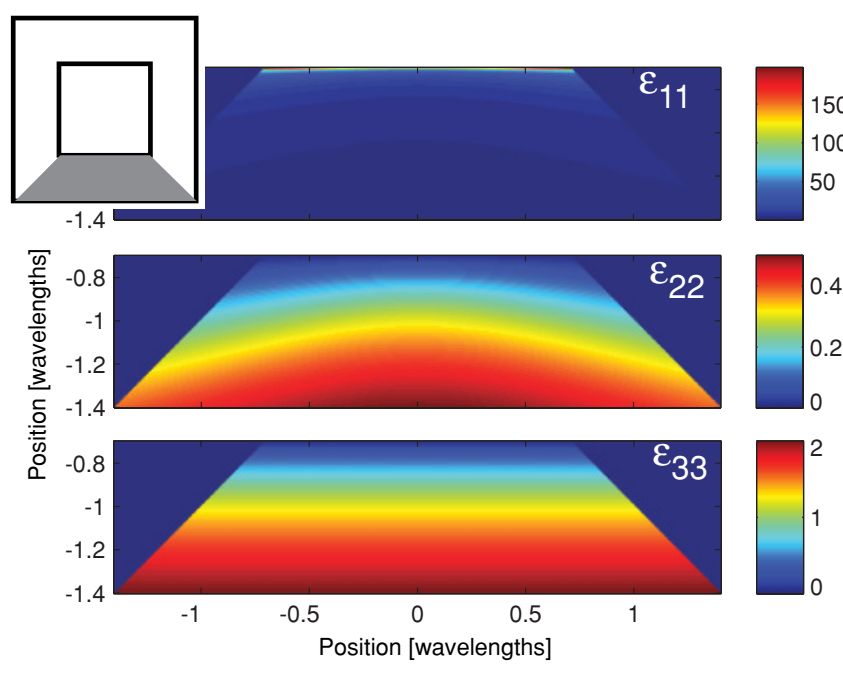

FIG. 1. (Color online) Material parameters inside the highlighted section of the square cloak shown in the inset, as obtained by Rahm et al. [12]. Two components of the permittivity and permeability tensors have parabolic level sets, while the other component has linear level sets.

that map $\Gamma_{b}^{r}$ in the real space to a curve of identical shape in the virtual space, $\Gamma_{b}^{v}$. Here the superscript $v$ symbolizes virtual space, while $r$ represents the curve in the real domain. Outside $\Gamma_{b}^{r}$, that is, the outside frontier of the transformed region of space, we have $u=x, v=y, w=z$. Additional constraints are imposed by the application. For example, if we want to create the illusion that an object represented by curve $\Gamma_{a}^{r}$ in the real space looks like an object of different shape specified in the virtual space by $\Gamma_{a}^{v}$, the transformation functions have to map $\Gamma_{a}^{v}$ into $\Gamma_{a}^{r}$. As a special case, if we want to make the object invisible, we set $\Gamma_{a}^{v}$ to a point.

As pointed out before, there are an infinite number of mapping functions that satisfy the preceding constraints. In this article we show how to choose these functions in order to obtain layered designs. Once we have the transformation functions, the permittivity and permeability tensors inside the transformed region required in order to obtain the desired field behavior specified in the virtual space are given by, respectively [16],

$$
\overline{\bar{\epsilon}}^{r}=\overline{\bar{\mu}}^{r}=\frac{A \overline{\bar{\epsilon}}^{v} A^{T}}{\operatorname{det}(A)},
$$

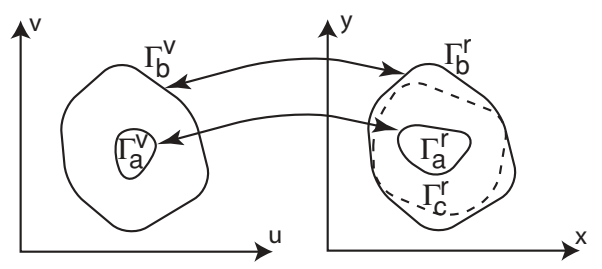

FIG. 2. Principle behind transformation optics. The interior of $\Gamma_{b}^{v}$ in the $(u, v)$ space (virtual space) transforms into the interior of the $\Gamma_{b}^{r}$ curve in the $(x, y)$ space (real space). The $\Gamma_{b}$ curves have the same shapes and sizes in both spaces; only their interior is stretched differently. where $\overline{\bar{\epsilon}}^{v}$ is the relative permittivity tensor in the virtual space $\left(\overline{\bar{\epsilon}}^{v}=\overline{\bar{\mu}}^{v}\right)$, and $A$ is the Jacobian matrix

$$
A=\frac{\partial(x, y, z)}{\partial(u, v, w)}=\left[\frac{\partial(u, v, w)}{\partial(x, y, z)}\right]^{-1} \equiv\left(\begin{array}{ccc}
u_{x} & u_{y} & u_{z} \\
v_{x} & v_{y} & v_{z} \\
w_{x} & w_{y} & w_{z}
\end{array}\right)^{-1}
$$

where we use the notation $h_{s} \equiv \partial h / \partial s$ with $h \in\{u, v, w\}$ and $s \in\{x, y, z\}$.

We assume that the permittivity and permeability in the virtual space are both equal and scalar quantities; consequently, we assume throughout the article that $\overline{\bar{\epsilon}}^{v}=\overline{\bar{\mu}}^{v}=I_{3}$, where $I_{3}$ is the identity matrix. Since, as a result, the relative permeability and permittivity tensors are going to be identical, we only refer to the permittivity tensor throughout.

As mentioned previously, we focus here on 2D mappings in which the transformation functions can be written

$$
\begin{gathered}
u=u(x, y), \\
v=v(x, y), \\
w=w(z),
\end{gathered}
$$

where $z$ and $w$ are the out-of-plane directions in the real and virtual spaces, respectively. In order to maintain the $2 \mathrm{D}$ requirement that field quantities are invariant in the $z$ direction, in our analysis $u$ and $v$ are independent of $z$, and $w$ is independent of $x$ and $y$. The large majority of 2D transformation-optics designs use $w=z$; however, others have shown that nonidentity mapping in the out-of-plane direction can prove useful. For example, Hu et al. [14] showed that singularities in the material parameter tensors can be avoided by carefully choosing $w$. For this reason we allow $w$ to vary arbitrarily with $z$.

The 2D transformations given by Eq. (3) lead to the following relative material parameter tensors obtained from Eq. (1):

$\overline{\bar{\epsilon}}^{r}=\frac{w_{z}^{2}}{\operatorname{det}\left(A^{-1}\right)}\left(\begin{array}{ccc}\left|\overline{\mathbf{u}}_{y}\right|^{2} & -\nabla u \cdot \nabla v & 0 \\ -\nabla u \cdot \nabla v & \left|\overline{\mathbf{u}}_{x}\right|^{2} & 0 \\ 0 & 0 & \frac{\operatorname{det}^{2}\left(A^{-1}\right)}{w_{z}^{4}}\end{array}\right)$,

where we used the notation $\overline{\mathbf{u}}_{s} \equiv \hat{\mathbf{x}} u_{s}+\hat{\mathbf{y}} v_{s}$, with $s \in\{x, y\}$, $\nabla \equiv \hat{\mathbf{x}} \partial / \partial x+\hat{\mathbf{y}} \partial / \partial y,|\cdot|$ represents the norm of a vector, and the dot is the inner product between two vectors. We note that, in most cases, if the transformation is nonconformal, then $\nabla u \cdot \nabla v \neq 0$, and the tensor expressed in Eq. (4) has nonzero off-diagonal elements. As pointed out in [12], from a practical point of view we need to express the material parameters of Eq. (4) in the principal system in which all tensors are diagonal because these are the tensor components of a material that would be engineered to have the parameters specified by Eq. (4). This approach was taken by Schurig et al. [3] to demonstrate a cloaking device at rf frequencies. It is worth noting that, for 2D mappings the principal system is obtained by rotating the $(x, y, z)$ system around the out-of-plane $z$ axis; therefore, $\epsilon_{z z}^{r}$ is invariant to this rotation. 
The components of the diagonalized $\overline{\bar{\epsilon}}^{r}$ (we call them $\epsilon_{1}, \epsilon_{2}$, and $\epsilon_{z} \equiv \epsilon_{z z}^{r}$ ) are the eigenvalues of $\overline{\bar{\epsilon}}^{r}$ [17], and, consequently, are solutions of the characteristic equation of unknown $t$,

$$
\operatorname{det}\left(\overline{\bar{\epsilon}}^{r}-t I_{3}\right)=0,
$$

which can be expanded into

$$
\left[t-\frac{\hat{\mathbf{z}} \cdot(\nabla u \times \nabla v)}{w_{z}}\right]\left[t^{2}-t \frac{|\nabla u|^{2}+|\nabla v|^{2}}{\hat{\mathbf{z}} \cdot(\nabla u \times \nabla v)} w_{z}+w_{z}^{2}\right]=0,
$$

where $\hat{\mathbf{z}}$ is the unit vector in the out-of-plane direction.

We recognize immediately the tensor component in the out-of-plane direction,

$$
\epsilon_{z} \equiv \epsilon_{z z}^{r}=\frac{\operatorname{det}\left(A^{-1}\right)}{w_{z}^{2}}=\frac{\hat{\mathbf{z}} \cdot(\nabla u \times \nabla v)}{w_{z}} ;
$$

therefore, the in-plane components of the diagonalized permittivity tensor are solutions of the simplified characteristic equation

$$
t^{2}-t \frac{|\nabla u|^{2}+|\nabla v|^{2}}{\epsilon_{z}}+w_{z}^{2}=0 .
$$

We emphasize that the simplified characteristic equation was derived for a general 2D mapping and holds regardless of the shape of the transformed domain or boundary conditions. The equation is important because it gives interesting insights into the material parameters required by any device obtained using transformation optics.

For example, from our layered-design point of view, it tells that the product of the in-plane components of each material parameter tensor expressed in the principal coordinate system is independent of the in-plane coordinates $x$ and $y$ :

$$
\epsilon_{1} \epsilon_{2}=w_{z}^{2} \text {. }
$$

A direct consequence of this relation is that $\epsilon_{1}$ and $\epsilon_{2}$ have the same level sets, that is, same contours of constant value; therefore, they have same-level sets as $\epsilon_{1}+\epsilon_{2}$, a quantity that is found from Eq. (8) to be

$$
\epsilon_{1}+\epsilon_{2}=\frac{|\nabla u|^{2}+|\nabla v|^{2}}{\epsilon_{z}} .
$$

It follows that, in order to obtain a layered architecture, either the level sets of $\epsilon_{z}$ should be a subset of the level sets of $\epsilon_{1}+\epsilon_{2}$, or the level sets of $\epsilon_{1}+\epsilon_{2}$ should be a subset of the level sets of $\epsilon_{z}$ (or both). In other words, there should exist a scalar one-dimensional function $g_{1}: \mathbb{R} \rightarrow \mathbb{R}$ or a function $g_{2}: \mathbb{R} \rightarrow \mathbb{R}$ (or both) for which

$$
\begin{gathered}
g_{1}(\hat{\mathbf{z}} \cdot[\nabla u \times \nabla v])=|\nabla u|^{2}+|\nabla v|^{2}, \quad \text { or } \\
g_{2}\left(|\nabla u|^{2}+|\nabla v|^{2}\right)=\hat{\mathbf{z}} \cdot(\nabla u \times \nabla v),
\end{gathered}
$$

where we used the expression of $\epsilon_{1}+\epsilon_{2}$ and $\epsilon_{z}$, as given by Eqs. (7) and (10). In the preceding expressions we neglected $w_{z}$, which is independent on $x$ and $y$. If both $g_{1}$ and $g_{2}$ exist, then $g_{1}$ is the inverse of $g_{2}$ and vice versa.

Equations (11) represent the most general condition for obtaining a layered transformation-optics device: We need to find $u(x, y)$ and $v(x, y)$ for which at least one of $g_{1}$ or $g_{2}$ exist. For example, conformal maps, that is, maps for which angles are preserved, satisfy the Cauchy Riemann equations $u_{x}=v_{y}$ and $u_{y}=-v_{x}$; therefore, both functions exist regardless of boundary conditions and $g_{1}(x)=2 x$ and $g_{2}(x)=x / 2$. Most transformation-optics devices, however, cannot be obtained from conformal maps. In this case, nonconformal transformations can be determined for which $g_{1}$ and/or $g_{2}$ still exist and consequently lead to layered devices.

\section{TRANSFORMATIONS BASED ON THE EIKONAL EQUATION}

Depending on the specific transformation-optics application, Eq. (11) can be further simplified. We focus here on the general class of invisibility shells depicted in Fig. 2, in which $\Gamma_{a}^{v}=\{(u, v) \mid u=0, v=0\}$; that is, the point of coordinates $(0,0)$ in the virtual space transforms to the $\Gamma_{a}^{r}$ curve that surrounds the object to be cloaked in the real domain. In this case it is suitable to choose the following family of mapping functions:

$$
\begin{aligned}
& u=x f(x, y), \\
& v=y f(x, y),
\end{aligned}
$$

where $f:$ annulus $\left(\Gamma_{a}^{r}, \Gamma_{b}^{r}\right) \rightarrow \mathbb{R}$ is a $2 \mathrm{D}$ scalar function defined on the transformed region in the $(x, y)$ space, that is, the region between the $\Gamma_{a}^{r}$ and $\Gamma_{b}^{r}$ curves in Fig. 2. The function $f$ should satisfy the following boundary conditions: $f\left(\Gamma_{a}^{r}\right)=0$ and $f\left(\Gamma_{b}^{r}\right)=1$ [throughout the article $f(\Gamma)$ represents a simplified notation for $f\left(x_{\Gamma}, y_{\Gamma}\right)$, where $\left(x_{\Gamma}, y_{\Gamma}\right)$ represents any point on the level set $\Gamma]$. The first condition specifies that $\Gamma_{a}^{r}$ in the real space maps to the point of coordinates $(u, v)=(0,0)$ in the virtual space. The second condition requires that $u=x$ and $v=y$ (i.e., identity mapping) at the outer boundary, $\Gamma_{b}^{r}$, of the transformed domain, as mentioned earlier.

The mapping given by Eq. (12) leads to the following simplified characteristic equation derived from Eq. (8):

$$
t^{2}-\left(2 w_{z}+\frac{|\overline{\mathbf{r}}|^{2}|\nabla f|^{2}}{\epsilon_{z}}\right) t+w_{z}^{2}=0,
$$

where $\overline{\mathbf{r}} \equiv \hat{\mathbf{x}} x+\hat{\mathbf{y}} y$ is the position vector. From this equation the condition for a layered design specified by Eq. (11) becomes (we recall that $w_{z}$ is independent of the in-plane coordinates $x$ and $y$ )

$$
\begin{gathered}
g_{1}\left(w_{z} \epsilon_{z}\right)=2 w_{z} \epsilon_{z}+|\overline{\mathbf{r}}|^{2}|\nabla f|^{2}, \quad \text { or } \\
g_{2}\left(2 w_{z} \epsilon_{z}+|\overline{\mathbf{r}}|^{2}|\nabla f|^{2}\right)=w_{z} \epsilon_{z} .
\end{gathered}
$$

An obvious choice for which both $g_{1}$ and $g_{2}$ exist (i.e., a layered architecture is achieved) is to choose $f$ so that

$$
|\overline{\mathbf{r}}||\nabla f|=k,
$$

where $k$ is a constant that will be determined shortly by imposing the boundary condition $f\left(\Gamma_{a}^{r}\right)=0$ and the condition that the level set characterized by $f=1$ is inside annulus $\left(\Gamma_{a}^{r}, \Gamma_{b}^{r}\right)$. In this case we have $g_{1}(x)=2 x+k^{2}$ and $g_{2}(x)=\left(x-k^{2}\right) / 2$. Comparing these functions to those computed earlier for conformal maps, that is, $g_{1}(x)=2 x$ and $g_{2}(x)=x / 2$, we note that Eqs. (12) and (15) always result in nonconformal mappings (we exclude here the uninteresting case $k=0$ which corresponds to the identity map). This is expected because 
full invisibility cloaks of the type presented in Fig. 2 cannot be implemented through conformal transformations [18]. The reason becomes clear if we look at the lines of constant $u$ and $v$ in the vicinity of the cloak inner boundary: Both these lines become parallel to the inner boundary and, therefore, do not maintain the $90^{\circ}$ angle between them required by conformal maps.

We recognize Eq. (15) to be the well-known eikonal equation, extensively used in optics to obtain light fronts propagating away from given sources. Since we impose the initial boundary condition $f\left(\Gamma_{a}^{r}\right)=0$, the cloak inner boundary, $\Gamma_{a}^{r}$, plays in this case the role of a source emitting a wave whose front propagates with speed $|\overline{\mathbf{r}}| / k$ variable with position. The function $f$ signifies the time needed by the front to reach a particular point $(x, y)$. Depending on the exact boundary geometry, the equation can be solved either analytically [19] or numerically using efficient algorithms analyzed extensively in the literature, such as the fast marching algorithm [20].

The constant $k$ can be determined from the boundary conditions using the following procedure. First we solve Eq. (15) for $k=1$ under the initial boundary condition $f\left(\Gamma_{a}^{r}\right)=0$, that is, find the function $f_{1}$ for which $|\overline{\mathbf{r}}|\left|\nabla f_{1}\right|=1$. Once we know $f_{1}$, we can determine its level set (we call it $\Gamma_{c}^{r}$ in Fig. 2) tangent to $\Gamma_{b}^{r}$. We require $f\left(\Gamma_{c}^{r}\right)=1$; therefore, given that $f=f_{1} k$, it follows that $k=f\left(\Gamma_{c}^{r}\right) / f_{1}\left(\Gamma_{c}^{r}\right)=1 / f_{1}\left(\Gamma_{c}^{r}\right)$. Finally, we set $f=1$ inside the region between $\Gamma_{b}^{r}$ and $\Gamma_{c}^{r}$. This assures the second boundary condition $f\left(\Gamma_{b}^{r}\right)=1$.

With $f$ determined we obtain the mapping functions from Eqs. (12). The material parameters of the layered device are then given by Eq. (4) in the $(x, y, z)$ coordinate system and by Eqs. (7) and (8) in the principal system.

\section{DESIGN OF A LAYERED SQUARE CLOAK}

As an example, we redesigned the square cloak considered by Rahm et al. [12] (see Fig. 1) in order to obtain a layered architecture. The curve $\Gamma_{a}^{r}$ is a square of side $a=1.4 \lambda$ while $\Gamma_{b}$ is a square of side $b=2.8 \lambda$, where $\lambda$ is the wavelength at which we perform the simulation. We solve numerically the eikonal equation using the fast marching method. We obtain $k=3.53$ for which the curve $f=1$ is tangent to the outside boundary of the cloak. Some of the level sets of $f$ obtained in such a way are presented in Fig. 3(a) (the thick green closed curves). The level sets of the mapping functions $u$ and $v$ obtained from Eq. (12) are shown in the same figure using thin red and blue lines. For simplicity, we chose the off-plane transformation to be the identity mapping $w=z$. As mentioned earlier, once we obtained $u$ and $v$ we can compute the material parameter tensors $\left(\overline{\bar{\epsilon}}^{r}=\overline{\bar{\mu}}^{r}\right)$ using Eq. (4).

The resulting tensors are obtained in the principal coordinate system with the help of Eq. (7) and the simplified characteristic equation (8). Figure 4 shows a selected number of level sets (i.e., contours of constant value) of the diagonalized permittivity tensor (recall that the permeability and permittivity tensors are equal). For clarity, the space between adjacent contours is colored with the same shade of color. We notice that $\epsilon_{1}, \epsilon_{2}$, and $\epsilon_{z}$ have identical level sets, as expected, which validates the design methodology. We also notice that toward the outer edge of the transformed region the level sets
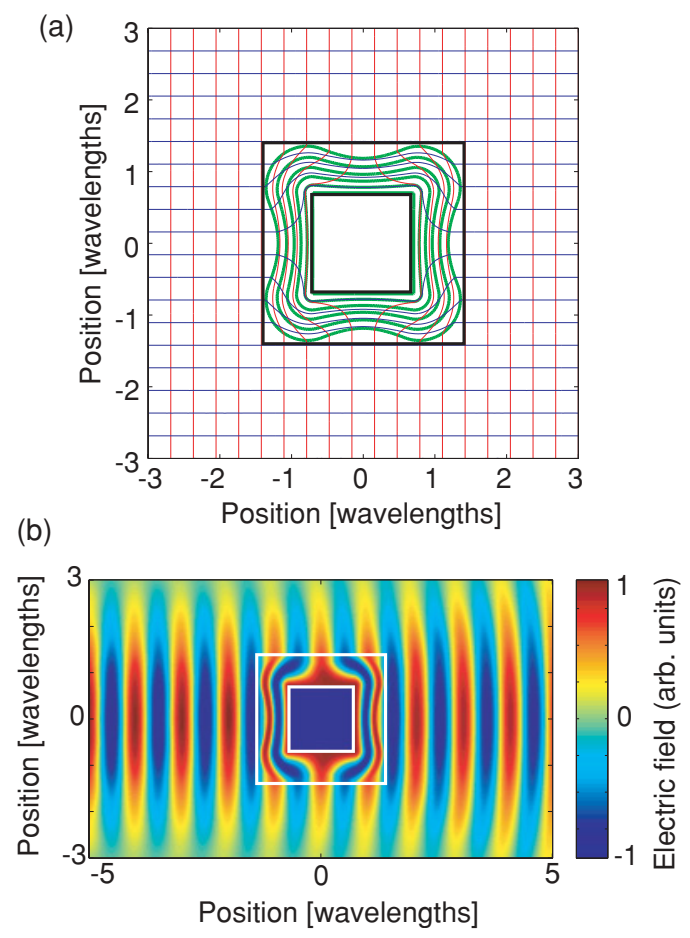

FIG. 3. (Color online) Transformations that produce a layered square cloak. The thick green curves represent level sets of function $f$. The thin blue (horizontal outside the cloak) and red (vertical outside the cloak) curves represent transformations of lines of constant $v$ and, respectively, $u$.

consist of a collection of closed curves, as opposed to those close to the cloaked object that are made of single curves. This layered geometry is unlike other cylindrical and spherical layered geometries of cloaking devices published in the past. A simulation of a Gaussian beam incident on the cloaked object is presented in Fig. 3(b) and shows minimal scattering, which confirms the cloaking behavior of our design.

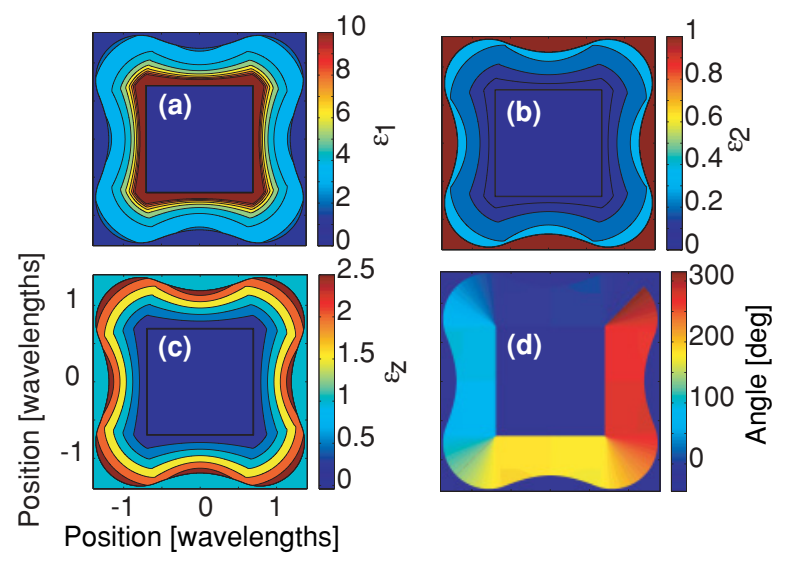

FIG. 4. (Color online) Material parameters inside layered cloak. (a) $\epsilon_{1}=\mu_{1}$; (b) $\epsilon_{2}=\mu_{2}$; (c) $\epsilon_{z}=\mu_{z}$. All three material parameters have level sets of same shape. The space between contour lines of constant value featured in the figure is colored using a single color shade in order to highlight the shape of the contour lines; (d) Rotation angle of principal system relative to $(x, y, z)$ coordinate system. 


\section{CONCLUSIONS}

We have presented an algorithmic approach to designing transformation devices of arbitrary shape that have a layered architecture. The advantage of such an architecture is that it is suitable to be implemented using the techniques extensively analyzed in the literature for cylindrical and spherical geometries. Through the development of the method we revealed some of the properties the material parameters inside 2D transformation devices have to satisfy [see Eqs. (7), (9), and (10)]. Based on these properties we determined general conditions for obtaining layered devices. Remarkably, a possible set of mapping functions that satisfy these conditions is derived directly from solutions to the well-known eikonal equation. This approach is general in that it can be applied to any type of transformation device (not only the invisibility cloaks) and any geometry.
[1] J. B. Pendry, D. Schurig, and D. R. Smith, Science 312, 1780 (2006).

[2] U. Leonhardt, Science 312, 1777 (2006).

[3] D. Schurig, J. J. Mock, B. J. Justice, S. A. Cummer, J. B. Pendry, A. F. Starr, and D. R. Smith, Science 314, 977 (2006).

[4] S. A. Cummer, B.-I. Popa, D. Schurig, D. R. Smith, and J. B. Pendry, Phys. Rev. E 74, 036621 (2006).

[5] H. Chen, B.-I. Wu, B. Zhang, and J. A. Kong, Phys. Rev. Lett. 99, 063903 (2007).

[6] W. Cai, U. K. Chettiar, A. V. Kildishev, V. M. Shalaev, and G. W. Milton, Appl. Phys. Lett. 91, 111105 (2007).

[7] Y. Huang, Y. Feng, and T. Jiang, Opt. Express 15, 11133 (2007).

[8] H. Ma, S. Qu, Z. Xu, and J. Wang, Opt. Express 16, 15449 (2008).

[9] R. Weder, J. Phys. A 41, 065207 (2008).

[10] B.-I. Popa and S. A. Cummer, Phys. Rev. A 79, 023806 (2009).

[11] C. L. Scandrett, J. E. Boisvert, and T. R. Howarth, J. Acoust. Soc. Am. 127, 2856 (2010).
[12] M. Rahm, D. Schurig, D. A. Roberts, S. A. Cummer, D. R. Smith, and J. B. Pendry, Photonics Nanostruct. 6, 87 (2008).

[13] W. X. Jiang, J. Y. Chin, Z. Li, Q. Cheng, R. Liu, and T. J. Cui, Phys. Rev. E 77, 066607 (2008).

[14] J. Hu, X. Zhou, and G. Hu, Appl. Phys. Lett. 95, 011107 (2009).

[15] Y. Lai, J. Ng, H. Y. Chen, D. Z. Han, J. J. Xiao, Z.-Q. Zhang, and C. T. Chan, Phys. Rev. Lett. 102, 253902 (2009).

[16] D. Schurig, J. B. Pendry, and D. R. Smith, Opt. Express 14, 9794 (2006).

[17] W. M. Lai, E. Krempl, and D. Rubin, Introduction to Continuum Mechanics, 4th ed. (Butterworth-Heinemann/Elsevier, Boston, MA, 2010).

[18] J. Li and J. B. Pendry, Phys. Rev. Lett. 101, 203901 (2008).

[19] L. C. Evans, Partial Differential Equations (American Mathematical Society, Providence, RI, 1998).

[20] J. A. Sethian, Level Set Methods: Evolving Interfaces in Geometry, Fluid Mechanics, Computer Vision, and Materials Science (Cambridge University Press, Cambridge, NY, 1996). 